\title{
COMPARISON OF THE EFFECTS OF TWO PUSH-TO-TALK BUTTON IMPLEMENTATIONS ON DRIVER HAND POSITION AND VISUAL ATTENTION
}

\author{
Oskar Palinko and Andrew L. Kun \\ University of New Hampshire \\ Electrical and Computer Engineering Department \\ Durham, New Hampshire, USA \\ E-mail: oskar.palinko@unh.edu, andrew.kun@unh.edu
}

\begin{abstract}
Summary: Buttons built into the steering wheel are used in many vehicles as push-to-talk (PTT) buttons for in-car speech user interfaces. We explore the influence of such a fixed PTT button on driver hand position on the steering wheel and on visual attention while driving. We also explore these variables for a wireless PTT glove, which allows drivers to use the entire surface of the steering wheel to operate the PTT button. Participants in our driving simulator-based study were willing to take advantage of the flexibility in hand position afforded by the glove PTT button. We also found that participants cast glances toward the steering wheel significantly less often when using the PTT glove than they did when operating the fixed PTT button.
\end{abstract}

\section{INTRODUCTION}

Many vehicles today have buttons built into the steering wheel. These buttons can be used to accomplish a diverse set of tasks, from adjusting the radio volume, to selecting a song on an MP3 player, to activating cruise control. With the proliferation of in-car speech user interfaces, steering wheel-based buttons are now also often used as push-to-talk (PTT) buttons. In prior research (Kun et al, 2007) we have shown that the usage of a fixed PTT button located on the center console may affect driving performance. In this paper we explore the effects of two onwheel PTT solutions on driver hand position on the wheel and on driver visual attention.

The two PTT solutions we explore are a fixed PTT button attached to the steering wheel and a wireless PTT glove. Our PTT glove (Palinko \& Kun, 2008) has two push buttons in its fabric, one under the thumb and one under the index finger. As the glove's integrated buttons are always under the driver's fingertips, we hypothesize that the glove PTT button will be operated in a wider range of hand positions than a fixed PTT button. We also hypothesize that this freedom to operate the button anywhere on the steering wheel will reduce the need for drivers to divert their visual attention from the road in order to locate the PTT button.

Of course, it is by no means clear that anybody would be willing to wear a glove while driving simply to gain the freedom to push the PTT button anywhere on the steering wheel. A better solution may be creating a steering wheel with a surface sensitive to the driver's taps. The taps could signal the beginning of an utterance as well as other control messages. However, instrumenting the entire surface of the steering wheel would be a challenging hardware task. The PTT glove uses simple hardware to sense actions that are similar to a user tapping on the steering wheel. Thus, we propose using the PTT glove to evaluate the concept of a steering wheel that has a surface sensitive to taps. 


\section{Related research}

Operating a fixed steering wheel button may be overwhelming in complex driving situations, like taking turns. In a pilot study we compared the usage of a fixed PTT button and a PTT glove while making turns (Palinko \& Kun, 2008). Participants reacted slower to a stimulus to operate the fixed PTT button than to a stimulus to operate the glove. Evidently, users wait to get out of a complex driving situation (making a turn) before reaching for the fixed button. Bergl et al. used steering wheel buttons to activate a voice recognition system for controlling cell phones (Bergl et al, 2006). They describe the system which is capable of name dialing, number dictation, adding contacts, etc. However, they do not concentrate on the effects of the buttons on aspects of driving as we do in this research. Sodnik et al. used steering wheel buttons to control a mobile device that produced either audio or visual feedback (Sodnik et al, 2008). A number of secondary tasks were performed on the mobile device while driving, such as changing the active user profile or deleting an image. Driving performance, mobile device task completion time, perceived workload and overall user satisfaction were measured. They found that the auditory feedback system is more efficient in most of the above measures except in task completion time. Again, this research did not evaluate the effects of using buttons on various aspects of driving. Gonzalez et al. investigated using a touchpad on the steering wheel to facilitate navigation related tasks such as selection from a list (Gonzalez et al, 2007). They found that gesture-based input is more efficient than keyboard-based text entry or direct list-selection methods. While the current implementation of our PTT glove uses push buttons, we plan to test a version of the glove with touch sensors.

Gloves are often used in research and development for gesture recognition, physiological and pressure measurements, robot control, etc. One commercial product was created by RallyPoint (RallyPoint, 2008) for military use, and it provides a multitude of control buttons that can operate a radio system, scroll through maps, make menu selections, etc.

A number of authors have dealt with interacting with in-car devices and the effects of these interactions on driving. An excellent overview of in-car speech user interface effects on driving can be found in (Barón \& Green, 2006). A recent paper investigates different user interfaces and their effect on eye fixations while driving (Bach et al, 2008).

\section{METHOD}

\section{Apparatus}

The hypothesis of this research was tested using the experimental approach. The participants operated a driving simulator while using a fixed push-to-talk button and the glove. We used a high-fidelity driving simulator (DriveSafety DS600c), shown in Figure 1. Its main features include: $180^{\circ}$ field of view, actual car cab, motion platform, realistic sounds and vibration. The driver's hand position on the steering wheel was recorded using a DV camcorder device. The video recording was later transcribed. Our driving simulator was equipped with an eye tracking system (SeeingMachines FaceLAB 4) mounted on top of the dashboard in front of the driver. Using the eye tracker, it was possible to record when the subjects were diverting their gazes to locate a PTT button on the steering wheel. 


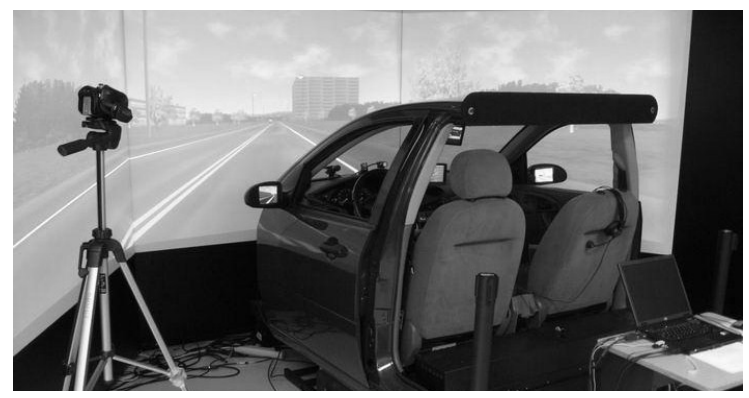

Figure 1. High-fidelity driving simulator and camera setup

The two PTT solutions are shown in Figure 2. Figure 2 a) shows the fixed PTT button. We used an AirClick computer remote controller by Griffin Technology, mimicking the built-in steering wheel buttons which come as standard equipment on many of today's vehicles. We told participants that all five buttons operated the PTT button. Figure $2 \mathrm{~b}$ ) shows the PTT glove. The glove was designed with momentary push-buttons under the fabric of the index finger and the thumb. An RF transmitter forwards the button-push information to a data collecting computer.

a)
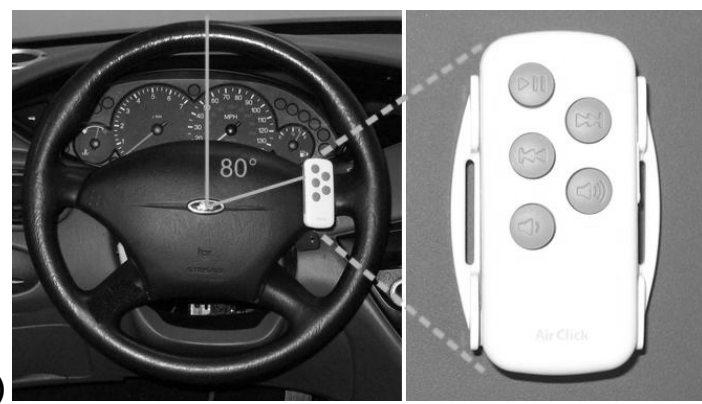

b)

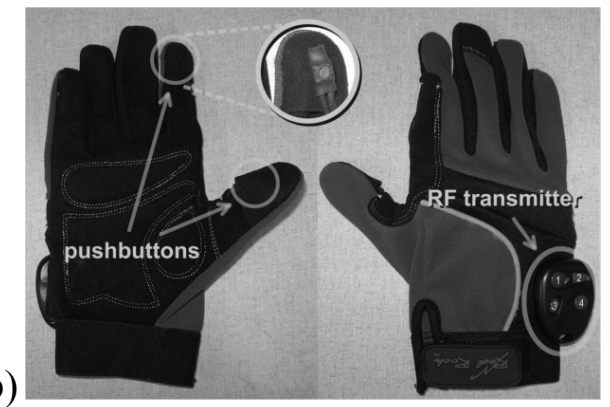

Figure 2. a) Fixed button device location and b) the PTT glove

\section{Participants}

There were 24 participants who completed the experiment. They were recruited on campus using e-mail newsletters and hallway posters. Most of the participants were college students or university staff. Their mean age was 26.04 years. There were 16 male and 8 female subjects. On average, they had around 8 years of driving experience. Two subjects were left-handed and 22 right-handed.

\section{Procedure}

The subjects were first given a personal information questionnaire. After that, they were introduced to the primary and secondary tasks of the experiment. Their primary task was to drive on a curvy two-lane rural highway with one lane in each direction. The curves were both left and right ones, with a radius of 230 meters. The subjects had to follow a lead vehicle travelling at 60 mph. They were told to follow the vehicle at a comfortable distance and not to lose sight of it. The secondary task was a command-and-control speech interaction task with the on-board computer system, which required the subjects to retransmit messages received over the radio system. This put the subjects in the role of a police officer. Participants were told about incoming messages and they had to issue commands to the radio system to forward these messages to the 
correct destination. Participants did not have to memorize the speech task grammar. Instead, the system prompts informed them of the appropriate utterances to use. The secondary task was performed in a Wizard of Oz style, i.e. the subjects thought that they were interacting with the on-board computer system, but the answers were actually provided by the experimenter. For a more detailed explanation of the tasks see (Palinko, 2008). The subjects trained for the primary and secondary tasks for around 25 minutes. After this they drove for another 25 minutes which was the recorded part of the experiment. During this time they used the fixed PTT button, the wireless PTT glove and ambient recognition (which did not involve any PTT signal) to issue commands. The interactions with the wizard also mimicked interactions with two different recognizers, one with a higher $(89 \%)$ the other with a lower $(44 \%)$ recognition rate. Half of the subjects used the push-release sequence of operating the PTT, while the other half used the pushhold-release sequence. In the push-release sequence participants pushed and released the PTT button to signal the beginning of an utterance, and then uttered the speech command. In the pushhold-release mode of operation participants held down the PTT button for the entire duration of an utterance. During the experiment we recorded participants' hand position on the steering wheel using a video camera. After finishing the experiment, participants filled out a questionnaire assessing their subjective opinions about the PTT buttons.

The study had two within-subject variables with their levels given in parentheses: PTT type (fixed, glove) and recognizer accuracy (high, low). Note that in this paper we do not report on data from participant interactions using ambient recognition (no PTT button). The study also had a between-subjects independent variable: PTT sequence (push-release, push-hold-release). In this paper we focus on the PTT type variable. The dependent variables considered here are: angular hand position on the steering wheel, number of visual fixations on the wheel and the mean length of fixations.

\section{Hand Position Coding}

For each participant the angular position of the hand on the steering wheel while operating the PTT button, or more precisely the angular position of the finger operating the PTT button, was transcribed from video recordings by one coder. Because of the limited precision of the recording, the angular positions were classified into $15^{\circ}$ bins. The bins were selected as follows: all values from $-7.5^{\circ}$ to $7.5^{\circ}$ were classified into the $0^{\circ}$ bin, $7.5^{\circ}$ to $22.5^{\circ}$ to the $15^{\circ}$ bin, and so on. The bins are shown in Figure 3 a). Figure 3 b) shows a still frame from the video recording, with the overlaid cabin coordinate system for determining angular position of the hand. The cabin coordinate system is fixed with respect to the cabin even as the steering wheel rotates.

This video overlaying technique was only used for encoding the position of the finger operating a PTT button on the glove. For the fixed PTT button the activating finger is always located at an $80^{\circ}$ angle in the coordinate system fixed to the steering wheel. Since steering wheel angles are provided by the simulator, we can readily find the hand position in cabin coordinates, by adding $80^{\circ}$ to the wheel angle. These angles can then be classified into the same $15^{\circ}$ bins as the glove PTT button angles. 
a)

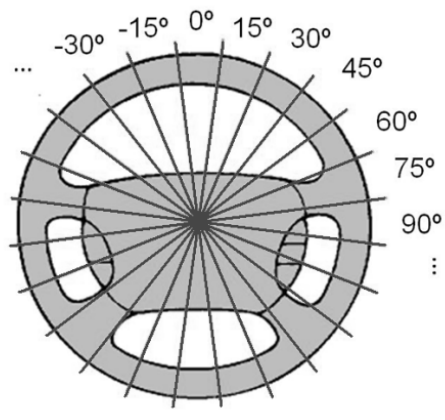

b)

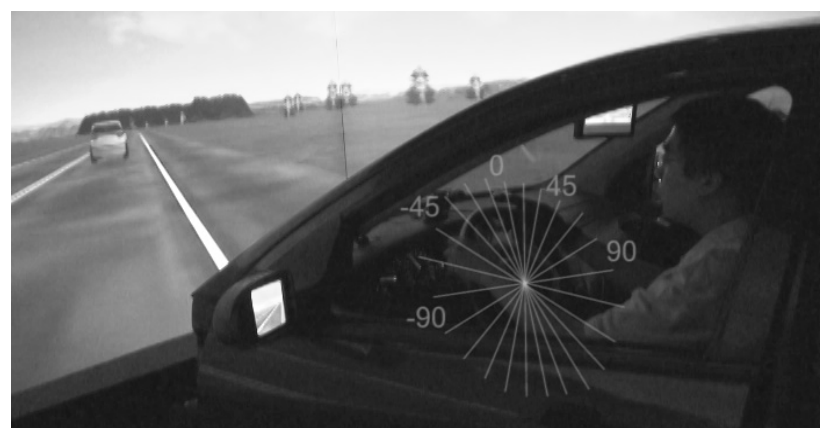

Figure 3. a) Steering wheel bins b) Bins overlaid with video

\section{RESULTS}

\section{Hand Position Data}

Figure 4 shows the number of activations of the PTT buttons for different driver hand positions. Driver hand positions are given in angles and are with respect to cabin coordinates. The dark bars represent glove positions and the light bars show fixed PTT activations. There is a clear distinction between the values for the glove and the fixed solution. Glove activations are more spread out than fixed PTT activations, and they follow a normal distribution with a mean around $45^{\circ}$. Fixed PTT activations come from data from three groups corresponding to driving in a left curve (peak at $45^{\circ}$ ), driving straight (peak at $75^{\circ}$ ) and driving in a right curve (peak at $105^{\circ}$ ).

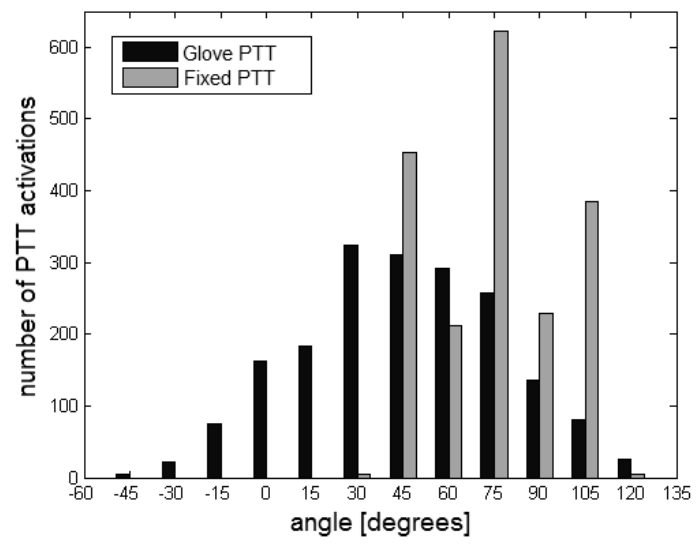

Figure 4. Histogram of hand angles in the cabin coordinate system

To be able to analyze these data using analysis of variance (ANOVA) we split the results into these three groups for both the fixed and glove buttons. After this classification the resulting histograms all resembled normal distributions, which allowed the ANOVA analysis. The analysis showed that the difference in angular position between the fixed and glove solutions is highly significant $(\mathrm{p}<0.0001)$, for all three driving situations. Also, the data for the glove is much more spread out than for the fixed solution. Clearly, when given a choice, drivers prefer to use, or are at least willing to experiment with using, a wider area of the steering wheel than afforded by the fixed PTT button. Also, they prefer to keep their hands and activate the PTT button around the " 2 o'clock" position on the steering wheel (30-45" in steering wheel coordinates), just 
as we were all taught in driver's education! This is of course different from the $80^{\circ}$ position in steering wheel coordinates, which was the location of our fixed PTT button, and which is roughly the location of many on-wheel buttons in cars.

\section{Visual Attention Data}

Any time spent not looking at the road ahead could potentially cause a safety threat, since an unexpected event can occur anytime. A delay in reaction to such an event can cause hazardous driving situations. When using the fixed PTT button participants often looked at the steering wheel, presumably searching for the button to push, just before operating the PTT button. We classified fixations at the steering wheel as being connected to PTT operation if they occurred within a 2 second window before PTT activation. Also, we only took into account fixations at parts of the steering wheel where the button was located, taking into account the fact that the button location changes with steering wheel motion. In the case of the glove subjects also cast glances toward the steering wheel, presumably as part of operating the PTT button, however they did so less often. Since the glove PTT buttons can be operated anywhere on the steering wheel we classified all fixations at the steering wheel that occurred within the 2 second time window before PTT operation as fixations connected to glove PTT button operation.

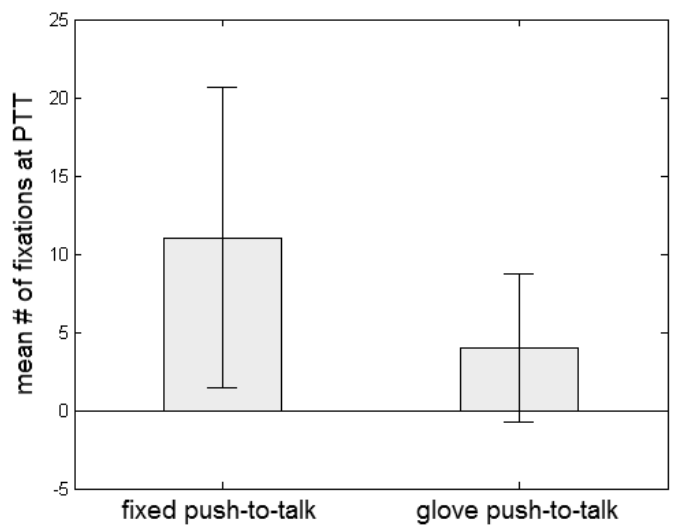

Figure 5. Mean number of fixations at the PTT during interactions

Figure 5 shows the number of fixations at the PTT during interactions, averaged over all subjects. We consider glances over 100 milliseconds in length to be fixations (Manor \& Gordon, 2003). The average number of fixations for all participants was 11.04 when using the fixed PTT button and 4.00 when using the glove. In both cases there were 54 button-pushes. The difference in the number of fixations was confirmed to be statistically significant using ANOVA $(\mathrm{p}<0.01)$. The mean length of fixations with the fixed PTT button was 0.31 seconds and with the glove it was 0.26 seconds. This difference in mean lengths was not statistically significant.

\section{CONCLUSION}

Our study confirmed the hypothesis that users will use the glove PTT button in a wider range of hand positions than they will use a fixed PTT button. We also confirmed the hypothesis that drivers are less likely to divert their eyes from the road when using the glove PTT button than they are when using the fixed PTT button. Of course, the safety implications of this latter finding are not clear. First of all, our simulated driving task was very simple. Participants followed a lead 
vehicle; there were no intersections, and no other complicating events. It is possible that in more complicated scenarios drivers would have been careful about the timing of operating the PTT button and would only have diverted their gaze from the road ahead when this was safe to do. In fact, our hypothesis is that this is not the case, but the evaluation of this hypothesis is left to a future study. Second, it is possible that after appropriate training participants would have been able to consistently find even the fixed PTT button without diverting their gaze from the road ahead. We are currently evaluating whether simply warning drivers that they should not look for the PTT button while driving can significantly impact the number of fixations on the fixed PTT button during driving. In practice such a warning could be included on sticker on the fixed PTT button to be removed when taking possession of a car at a dealership.

In our effort to use the glove to evaluate a steering wheel sensitive to taps, we plan to replace the push buttons on the PTT glove with touch sensors. These sensors will allow subjects to interact with a computer using taps as opposed to clicks afforded by the current version of the glove.

\section{ACKNOWLEDGEMENTS}

This work was supported by the US Department of Justice under grants 2005CKWX0426 and 2006DDBXK099. The authors would like to thank Željko Medenica for his help with the statistical analysis of the data.

\section{REFERENCES}

Bach K.M., Jaeger M.G., Skov M.B., Thomassen N.G. (2008). You Can Touch, but You Can’t Look: Interacting with In-Vehicle Systems. CHI.

Barón A., Green P. (2006). Safety and Usability of Speech Interfaces for In-Vehicle Tasks while Driving: A Brief Literature Review. UMTRI Technical Report 2006-5.

Bergl V., Cmejrek M., Fanta M., Labsky M., Seredi L., Sedivy J., Lubos U. (2006). CarDialer: Multi-Modal In-Vehicle Cellphone Control Application. Conf. of Multimodal Interfaces.

Gonzalez I.E., Wobbrock J.O., Chau D.H., Faulring A., Myers B.A. (2007). Eyes on the Road, Hands on the Wheel: Thumb-Based Interaction Techniques for Input on Steering Wheels. Graphics Interfaces.

Kun A.L., Paek T., Medenica Ž. (2007). The Effect of Speech Interface Accuracy on Driving Performance. Interspeech.

Manor B., Gordon E. (2003). Defining the Temporal Threshold for Ocular Fixation in FreeViewing Visuocognitive Tasks, Journal of Neuroscience Methods, Vol. 128, Iss. 1-2.

Palinko O. (2008). Effects of Different Push-To-Talk Solutions on Driving Performance. Master's Thesis, University of New Hampshire.

Palinko O., Kun A.L. (2008). Prototype Wireless PTT Glove. IET Conference.

RallyPoint Inc. (2008). Handwear Computer Input Device. http://www.rallypoint.info/docs/hcid.pdf, last visited December 1, 2008.

Sodnik J., Dicke C., Tomaič S., Billinghurst M. (2008). A User Study of Auditory Versus Visual Interfaces for Use While Driving. Int. Journal of Human-Computer Studies, Vol. 66, Issue 5. 